\title{
Ueber virulente Bubonen und den Ulcus molle-Bacillus.
}

Von

Dr. Rudolf Krefting, Christiania.

Was die Entwickelung von Bubonen nach Ulcus molle bedingt, ist bei der Mehrzahl der vorkommenden Fälle unaufgeklärt.

Die nicht virulenten enthalten bekanntlich Eiter, in welchem weder mikroskopisch noch durch Züchtungsversuche auf den bekannten Nahrungssubstraten Mikroben nachgewiesen werden können.

Bei Probeinoculationen gibt dieser Eiter keinen Ausschlag und entspricht dem, was man bakteriologisch unter sterilem Eiter verstehe.

Diese Art Bubonen sind der Erfahrung aller Kliniken gemäss die am häufigsten vorkommenden und können periodisch in so überwiegend grosser Anzahl vorkommen, dass einzelne Forscher, ${ }^{1}$ ) die eine Serie solcher Fälle angetroffen, sich haben verleiten lassen, die Existenz der virulenten Bubonen zu leugnen.

Einzelne Fälle von Drüseneiterung nach Uleus molle, wo Staphylococcen oder Streptococcen nachgewiesen werden können, müssen als durch Mischinfection hervorgerufen angesehen werden und dürfen nicht hierher gerechnet werden, obschon sie sich klinisch den nicht virulenten Bubonen mit sterilem Eiter sehr ähnlich verhalten. Was die virulenten Bubonen betrifft, scheint dagegen die Aetiologie klar zu sein, obwohl noch verhältnissmässig

1) Traedgaard, Strauss. Aus der späteren Zeit Ducrey. 
wenige Untersuchungen vorliegen, die das Vorhandensein vom Ulcus molle Bacillus in denselben zeigen.

Da es nur meine Absicht ist, ganz kurz meine fortgesetzten Untersuchungen der ron mir behandelten Fälle von Bubonen mitzutheilen, will ich die Geschichte mit Rücksicht auf die Ulcus molle-Frage nicht wiederholen. Seitdem ich mich mit der Frage beschäftigt habe, ist es mir oft eingefallen, dass Ulcus molle nicht ein so sicher begrenzter Krankheitsbegriff zu sein braucht, und dass die Krankheit z. B. nicht vollständig dieselbe ist, wenn sie in Christiania auftritt, wie in Neapel, Wien etc., sowie dass Finger, der angibt, dass die Wiener Schule den Krankheitsbegriff etwas weiter definirt, zum Theil Recht haben könnte.

Es liegen jedoch jetzt nach dem Nachweise des Bacillus so zahlreiche, völlig übereinstimmende Untersuchungen aus so vielen weit von einander liegenden Orten vor, dass die $\mathrm{Kr}$ an kheit überall als einartig und unbedingt dieselbe constatirtwerden muss. Die Anzahl derjenigen, die ihre Funde rom Bacillus in den Schankerwunden und den Inoculationspusteln mitgetheilt haben, ist schon jetzt sehr gross.

Der, welcher zuerst den Bacillus nachwies, Ducrey, war: aus dem südlichsten Theil Europas und kam mit seinen Untersuchungen im Jahre 1889. ${ }^{1}$ ) Darauf kamen meine Untersuchungen, die zum Theil von mir ausgeführt waren, ohne D u cr e y zu kennen, aus dem nördlichsten Theile Europas - Cbristiania - veröffentlicht im. Jahre 1891. ${ }^{2}$ ) Dann Unna ${ }^{3}$ ) aus Hamburg im Jahre 1892, der eine Methode zum Färben der Bacillen im Schnitt erfand. Quinquaud und Nicolle ${ }^{4}$ ) aus Paris, Petersen ${ }^{5}$ ) aus St. Petersburg, Riviere ${ }^{6}$ ) aus Frankreich

1) Congres internat. de dermat. et de syph. Paris 1889. Comptes rendus p. 279.

$\left.{ }^{2}\right)$ Nord. med. Archiv 1891. Archiv für Derm. u. Syph. 1892. Ann. de derm. et syph. 1893.

3) Monatshefte für prakt. Dermat. 1892 u. 1895.

4) Annales de Dermatol. et syph. 1892 p. 818. Thèse de Paris 1893.

s) Wratsch. Februar 1893.

i) Journal des connaissances méd. Mai 1893. 
Walther Petersen ${ }^{1}$ ) aus Bonn, Mermel, ${ }^{2}$ Colombini ${ }^{3}$ ) aus Siena, A u dry ${ }^{4}$ ) aus Toulouse, Dubreuilh und Lasnet ${ }^{3}$ ) aus Bordeaux, Spietschka ${ }^{6}$ ) aus Prag. Aus Wien und Breslau haben Rille ${ }^{7}$ ) und Buschkas) Untersuchungen mit positivem Resultat mitgetheilt.

Bei einem Falle von Uleus molle auf der Zunge ist in Paris in Fournier's Abtheilung der Bacillus sowohl im Secret der Wunde als auch in den Wundwänden nachgewiesen worden. ${ }^{\mathbf{9}}$ )

Bei einem Falle von Ulcus molle am Finger, der als Panaritium angenommen war, hat der Nachweiss vom Ulcus molle-Bacillus die Diagnose bestätigt. ${ }^{10}$ )

Wenn man sieht, dass alle diese Untersuchenden aus so fern von einander liegenden Orten im Wesentlichen zu demselben Resultat gekommen sind, kann man sich nicht - wie Verfasser neuerer Lehrbücher es thun - allzu skeptisch verhalten, selbst wenn es auch noch Niemandem gelungen ist, die Mikroben auf künstlichen Nahrungssubstraten zu zuichten.

Eine Art Reincultur ist es jedoch, wenn man den Bacillus in Serien von Inoculationspusteln ohne Beimischung von anderen Mikroben nachweisen kann - eine Reincultur mit der menschlichen Haut als Nahrungssubstrat. Der Leprabacillus kann weder gezüchtet noch inoculirt werden, aber dennoch zweifelt Niemand daran, dass er die Krankheit hervorruft.

Während in Betreff der Schankerwunden zahlreiche, völlig übereinstimmende Untersuchungen, die alle das Vorhandensein des Bacillus sowohl im Eiter aus denselben in den Wundwänden (Schnitt) und den Inoculationspusteln, bestätigen, sind die Mittheilungen, welche ïber dessen Verhältniss zu den Bubonen

1) Centralbiatt f. Bakter. Juni 1893.

$\left.{ }^{2}\right)$ Archiv gen. de med. August 1893.

3) Comm. clin. delle mal, cut. e gen. 1893-94.

4) Gazetíte hebdom. 1893.

¡) Archiv klin. de Bordeaux. 1893.

6) Archiv f. Derm. u. Syph. 1894.

7) Verhandl. V. Deutscher Dermat.-Congress in Graz. 1895.

$\left.{ }^{8}\right)$ Ebendaselbst. Ref. in diesem Archiv 1896.

$\left.{ }^{9}\right)$ Emmery und Sabourand. Annales de dermat. p. 198. 1896.

${ }^{10}$ Krefting. Extragenitale Ulcera mollia. 7 Fälle. Norsk. Nag. for Laegev. 1896 p. 158. 
vorliegen, ziemlich spärlich. Was die nicht virulenten $\mathrm{Bub}$ on en betrifft, sind jedoch alle Untersucher darüber einig, dass in diesen weder Ulcus molle-Bacillen noch andere Mikroben nachgewiesen werden können.

Nachweis vom Ulcus molle-Bacillus in 2 Fällen von virulenten Bubonen sind von mir schon im Jahre 1892 in diesem. Archiv mitgetheilt worden.

Der eine dieser Fälle war besonders merkwürdig, indem bei demselben Patienten sich in der einen Leiste ein virulenter und in der anderen ein nicht virulenter Bubo befand.

In den „Annales de derm. et syph. 1893 pag. 837" wurde ein dritter Fall mitgetheilt, woselbst ich ausser im Buboeiter und den hiermit hervorgerufenen Inoculationspustein, nach Unna's Methode die Bacillen auch in Schnittpräparaten von excidirten. Stücken der Bubowand, nachwies. In demselben Jahre kamen Dubreuilh und Lasnet's ") Untersuchungen einer grossen Anzahl von Bubonen während einer Ulcus molleEpidemie in Bordeaux. Die Genannten kamen ebenfalls zu einem positiven Resultat und schreiben die Virulenz dem Vorhandensein des "bacille de Ducrey" zu.

"Von dem Augenblick an, da der Bacillus nachgewiesen werden kann, ist der Eiter virulent und inoculabel."

In den nicht virulenten Bubonen fanden diese Forscher auch keine Mikroben.

In einer kurzen Zeit - vom 12. Januar bis 11. August 1893 - haben dieselben observirt:

136 Fälle von Bubonen, von denen 27 resorbirt wurden, ohne sich zu öffnen. 43 , die geöffnet wurden, waren nicht virulent. 51 wurden nach dem Oeffnen virulent, waren es aber nicht gleich. 12 Fälle, die sich von selbst geöffnet hatten, ehe die Patienten ins Hospital gebracht wurden, waren sämmtlich virulent. 3 Fälle waren virulent vom ersten Tage an.

Es scheint eine sehr intensive Epidemie gewesen zu sein, sowohl mit häufig rorkommenden Fällen von Bubonen als auch intensiven Fällen, indem von 109 Fällen, die sich öffneten oder geöfinet wurden, 69 virulent waren.

2) Archives cliniques de Bordeaux. 1893. Nr. 10 und 11. 
Spietschka aus Prag, der in diesem Archiv im Jahre 1894 eine Reihe Untersuchungen von Bubonen, 46 Fälle, mitgetheilt hat, hat nur $2 \mathrm{Mal}$ gesehen, dass der Bubo schankrös wurde und beide Male nach dem Oeffnen. Sowohl im Wundsekret als auch in den hiermit hervorgerufenen Inoculationspusteln konnten die Bacillen nachgewiesen werden. Der Verfasser legt jedoch diesen beiden Fällen keine Bedeutung bei, da er die Möglichkeit nicht ausschliessen kann, dass eine von Aussen kommende Infection die Ursache ihres Schankröswerden gewesen sein kann.

Ich möchte jedoch geneigt sein zu glauben, dass dies nicht der Fall gewesen ist, da es nicht so selten geschieht, dass die Virulenz und damit der Bacillus erst nach dem Oeffnen des Bubos ${ }^{1}$ ) auftritt, welches auch mit Dubreuilh's Untersuchungen stimmt. Für D u breuilh ist es Regel gewesen, dass der Bubo im Augenblicke des Oeffnens nicht virulent war. Nur 3 Mal fand er "virulence d'emblee".

Wenn man im Uebrigen die Fälle von Dubreuilh, wo über die Hälfte virulent war, und Spiets chka's 46 Fälle, von denen nur 2 virulent wurden, vergleicht, bekommt man den Eindruck, dass das Virus in Bordeaux sich am Intensität sehr von dem Virus in Prag unterscheiden musste.

Jeder, der Inoculationsversuche vorgenommen hat, wird auch erfahren haben, dass der Anschlag in Bezug auf Intensität bedeutend variiren kann, gleichwie man erfahren haben wird, dass die Bacillen in grösster Menge vorhanden sind, wenn der Anschlag lebhaft ist. $\left.{ }^{2}\right)$ Wenn die Angaben mit Rücksicht auf die Häufigkeit der Bubonen, zumal der virulenten Bubonen, aus den rerschiedenen Städten so abweichend sind, muss man annehmen, dass dies ron Abweichungen im Charakter der Epidemien herrührt.

1) In meiner, im Jahre 1892 in diesem Archiv mitgetheilten Arbeit habe ich etwas ühereilt angenommen, dass ein von Anfang an nicht virulenter Bubo nicht virulent wird, ohne dass er von aussen inficirt wird. Später habe ich selbst Gelegenheit gehabt, einen Fall zu beobachten, wo die Virulenz erst 3 Tage nach dem Oeffnen eintrat.

2) "Die Menge der Bacillen steht im Verhältniss zur Intensität des Ausschlags." Krefting. Annales de dermat. et syph. 1893 p. 838. 
Aus Breslau liegen jetzt auch Untersuchungen von virulenten Bubonen mit positivem Resultat von $B$ uschke ${ }^{1}$ ) vor, der in 9 ron den untersuchten 36 Fällen die Bacillen fand, die er auch in Schnittpräparaten vom Düsenparenchym nachweisen konnte.

In sämmtlichen 9 Fällen wạr der Eiter im Augenblicke des Oeffnens inoculabel, aber der Verfasser gibt zu, dass es Fälle gibt, wo der Bubo erst melrere Tage nach dem Oeffnen schankrös wird und erklärt es auf die Weise, dass die im Eiter vorhandenen Bacillen todt sind, dass aber das in der Tiefe noch nicht zerfallene Drüsengewebe die virulenten Mikroben enthält, die das Schankröswerden veranlassen. $\mathrm{R}$ aff, ${ }^{\mathbf{2}}$ ) auch aus Breslau, berichtet von Jadas s o hn's Abtheilung von 22 Fällen von Bubonen nur $2 \mathrm{~F}$ älle virulenter Bubonen, die dasselbe Verhältniss wie Buschke's 9 Fälle zeigten. "In den übrigen 20 Fällen ergab sowohl die mikroskopische als auch die bakteriologische Untersuchung ein negatives Resultat (steriler Eiter).

In Wien fand Rille ${ }^{3}$ ) von 100 Fällen 9 mit inoculablem Eiter, mit Bacillen, die auch in excidirten Drüsenstïcken und excidirten Impfgeschwüren nachgewiesen werden konnten. Er bekam auch stets Anschlag mit Materie, die unmittelbar nach dem Oeffnen genommen worden.

Im Uebrigen hat Rille eine Statistik der Anzahl der Haftungen nach Impfungen mit Buboeiter gesammelt.

Unter 2117 Inoculationen, von 22 Autoren vorgenommen, befinden sich 609 Haftungen, also $28 \%$.

Im Anschluss an diese verhältnissmässig wenigen vorliegenden Mittheilungen über den Fund des Ulcus molle-Bacillus in virulenten Bubonen will ich in Kürze meine fortgesetzten Untersuchungen erwähnen, die übrigens nicht Neues ergeben, sondern nur bestätigen, was früher mitgetheilt worden. In den Jahren 1895 und 1896 habe ich unter meiner

1) Verhandl. des V. deutschen Dermatologen-Congresses Graz. 1895. Ref. in diesem Archiv 1896. XXXIV. 24.

2) V. Dermat.-Congr. In diesem Archiv Bd. XXXIV p. 121.

3) Daselbst. 
Privatclientel 143 Fälle von Ulcus molle behandelt, wovon 27 Fällemit Bubonencomplicirtwaren - also nur $19 \%$.

Ich habe hier nur die Fälle mitgerechnet, die geöffnet werden mussten. Sämmtliche Fälle wurden, mit Ausnahme eines virulenten Falles, wo der Patient wegen hochgradiger Schmerzen einige Tage zu Bette lag, a mbulatorisch behandelt und die Patienten gingen mit Stärkebandagen herum. Unter diesen 27 Fällen waren 7 virulent mit inoculablem Eiter.

WennVirulenz vorbanden war, wurde nie vergeblich nach Bacillen gesucht.

In einem dieser 7 Fälle trat die Virulenz erst 3 Tage nach dem Oeffnen auf.

Im Uebrigen bieten diese Fälle nichts von dem obenerwähnten Abweichendes dar. Die Zahlen sind leider allzu klein, als dass es eine Bedeutung haben könnte, den Procentsatz der Virulenz auszurechnen, der hier $26 \%$ war.

Diese Zahl entspricht jedoch ziemlich genau der Durchschnittszahl, welche Rille ron alier rorliegenden Inoculationsstatistik ausgerechnet hat, nämlich $28 \%$.

Die übrigen 20 Fälle enthielten sterilen Eiter, es konnten weder durch mikroskopische Untersuchungen noch durch Züchtungsversuche Mikroben nachgewiesen werden. Ich will den Platz hier nicht zu sehr in Anspruch nehmen, in dem in diese meine Fälle näher referire, aber diese, sowie früher beobachtete haben mich dazu gebracht, einige allgemeine Betrachtungen, sowie einen Vergleich zwischen virulenten und nicht virulenten Bubonen rom klinischen Standpunkt anzustellen.

\section{Weshalb bekommt man Bubonen nach Ulcus molle?}

Obschon es von besonderer Bedeutung für die Prophylaxe der Bubonen sein miisste, hierüber Gewissheit zu bekommen, muss doch diese Frage leider dahin beantwortet werden, dass man den Grund nicht kennt. Freilich werden verschiedene Gelegenheitsursachen angegeben, aber dieselben spielen keine entscheidende Rolle. 
Gleichwie man beobachten kann, dass die hartnäckigsten und virulenten Bubonen sich bei einem Patienten entwickeln können, der ganz ruhig in einem Hospital liegt, sieht man auch, dass keine Bubonen bei Patienten mit Ulcus molle kommen, die gehen, stehen und den ganzen Tag hart arbeiten.

Dass die Bildung von Bubonen von der Grösse der ursprünglichen Wunden nicht abhängt, geht aus meinen Fällen hervor. Ich habe gerade hierauf meine Aufmerksamkeit gerichtet und gefunden, dass die ursprünglichen Wunden in der Regel verhältnissmässig klein und wenig umsichgreifend sind und verhältnissmässig schnell zuheilen in den meisten Fällen, wo sich Bubonen entwickeln.

In einem dieser meiner Fälle von virulenten Bubonen batte ich die ursprüngliche Schankerwunde exstirpirt. Die Operationswunde heilte pr. primam und dennoch entwickelte sich ein virulenter Bubo.

In einem anderen Falle entwickelte sich ein virulenter Bubo 14 Tage nach dem Zuheilen der ursprünglichen Wunde. Solche Fälle werden von vielen erwähnt.

Bei den meisten der ron mir beobachteten Fälle haben die ursprünglichen Wunden ihren Sitz am Frenulum gehabt und dasselbe perforirt und abgefressen. Vielleicht ist das Frenulum eine Localisation, von der aus es dem Lymphstrom am leichtesten fällt, die Bacillen nach den Leistendrüsen zu führen. Das Frenulum als die der Infection am meisten ausgesetzte Stelle ist jedoch so oft der Sitz weicher Schankerwunden, ohne dass Bubonen kommen, dass auch dieses Verhältniss nicht erklären kann, weshalb die Bubonen kommen.

Von den 143 Fällen von Ulcera mollia aus meiner Privatpraxis, die ich hier erwähnt habe, waren nur 27 Fälle mit Bubonen complicirt - ca. $19 \%$.

Berichte aus Hospitälern zeigen selbstredend einen viel grösseren Procentsatz von Bubonen im Verhältniss zu den behandelten Fällen von Ulcus molle, da rorzugsweise die complicirten Fälle das Hospitel aufsuchen.

Nach Du breuilh ist ca. die Hälfte der im Hospital behandelten Fälle mit Bubonen complicirt. Jullien hat eine 
Statistik über 2698 Fälle von Ulcus molle, wovon $57 \%$ mit Bubonen complicirt waren.

Petersen in St. Petersburg hat eine Statistik über 4275 Fälle mit einer Häufigkeit von Bubonen von $39 \%$.

Neumann hat auf 2696 Fälle von Ulcus molle $41 \%$ mit Bubonen complicirt. Ungefähr denselben Procentsatz hat $\nabla$. Zeissl. Ebensowenig wie man die Frage, weshalb überhaupt Bubonen kommen, beantworten kann, ebenso wenig kann man erklären, weshalb einige Bubonen virulent werden, während der grösste Theil nicht virulent wird, ungeachtet die ursprünglichen Wunden in sämmtlichen Fällen keinen Unterschied zeigen.

Eins scheint jedoch sicher zu sein: damit Virulenz zu Stande kommen soll, muss der Ulcus molle-Bacillus die Lymphbahnen bis zu den Drüsen passiren.

Was dagegen den sterilen, nicht inoculablen Eiter in den nicht virulenten Bubonen hervorruft, weiss man nicht. Bis auf Weiteres muss man sich mit der Hypothese über die Stoffwechselproducteder Mikroben, die Eiterung obne Bakterien hervorrufen können sollen, begnügen.

\section{Die Häufigkeit der virulenten Bubonen.}

Die Angaben über die Häufigkeit der virulenten Bubonen im Verhältniss zu den nicht virulenten, variirt, wie man sieht, bedeutend.

Während Dubreuilh aus Bordeaux, wenn nur die geöffneten Bubonen mitgerechnet werden, $68 \%$ virulente hat, sind die Angaben aus Breslau, wenn man Buschke und Raff's Fälle zusammen legt, $19 \%$. Aus Prag nach Spietscha nur $4 \%$. Aus Wien nach Rille nur $9 \%$. Nach meinen Fällen von früher und jetzt sollte das Verhältniss ca. $25 \%$ sein.

Ich habe mit Willen hier nur Untersuchungen mitgenommen, die nach der Entdeckung des Bacillus vorliegen.

Wie man sehen wird. sind diese Zahlen bedeutenden Schwankungen unterworfen, nicht nur an den rerschiedenen Orten, sondern auch in derselben Stadt, indem Buschke und Raff aus Breslau resp. $25 \%$ und $8 \%$ haben. 
Wenn das Verhältniss mit Bezug auf die Virulenz so bedeutend variiren kann, ist es auch nicht so seltsam, dass einzelne Untersucher, die eine Serie von nicht virulenten $\mathrm{Bu}-$ bonen treffen, sich dazu verleiten lassen können, die Existenz der virulenten Bubonen zu leugnen. Im Allgemeinen sind die virulenten Fälle nicht gleichmässig vertheilt zwischen den nicht virulenten, sondern sie kommen nacheinander.

Wenn die Abweichungen mit Rücksicht auf die Häufigkeit der Virulenz nicht recht bedeutend sind, so kann dies ja nur auf einem Zufall beruhen.

Die Untersuchungsserie ist nicht grossgenug gewesen; aber wenn die Abweichungen so gross sind wie zwischen Dubreuilh's $68 \%$ aus Bordeaux und Spietschka's 4"\% aus Prag, muss man annehmen, dass dieses gewissen Verhältnissen beim Virus zuzuschreiben ist, die der Epidemie ihr Gepräge geben dürften.

Als Ausdruck eines beständigen $A b-$ und Zunehmens in der Intensität des Virus kann das bekannte periodenweise, plötzlich epidemische Auftreten der Krankheit in verschiedenen Städten ${ }^{1}$ ) genannt werden, während sie zu anderen Zeiten beinahe aussterben kann. Während die Anzahl der Fälle von Syphilis und Gonorrhoe freilich bedeutend wechseln kann, sind diese Krankheiten doch nicht solchen plötzlichen Schwingungen unterworfen wie Ulcus molle.

Man hat gesagt, dass die grössere oder geringere Häufigkeit von Ulcus molle ein besonderer Indicator für eine schlechte oder gute Controle sein sollte. Dies ist auch bis zu einem gewissen Grade richtig, insofern als diese Krankheit sich verhältnissmässig leicht controliren lässt, aber es sind doch auch gewiss andere unbekannte Verhältnisse in Betreff der Intensität und Lebensfähigkeit des Virus vorhanden, die eine grosse Rolle spielen und von der Controle unabhängig sind. Hier aus Christiania hat W. Boeck unter seiner Syphilisationsbehandlung gute Beweise dafür geliefert, wie das Virus förmlich aussterben

1) Ulcus molle scheint besonders epidemisch aufzutreten in Seestädten, wo alsdann besonders die Seeleute anscheinend neuen und frischen Virus von dem einen Ort zum andern bringen. 
konnte - es verlor seine Virulenz - selbst wenn es fortwährend von einem Individuum auf andere geführt wurde.

Wenn der Christianiafjord durchs Eis geschlossen war und keine Fahrzeuge kommen und mit ihnen Seeleute mit neuem Import ron Ulcera mollia, konnte es geschehen, dass es unmöglich war, Virus in der Stadt aufzutreiben.

\section{Der klinische Unterschied zwischen nicht virulenten und virulenten Bubonen.}

Ich will diese kurze Mittheilung wicht schliessen ohne den grossen klinischen Unterschied zwischen virulenten und nicht virulenten Bubonen, sowohl mit Rücksicht auf Symptome als auch Verlauf, zu pointiren. Schon ehe der Bubo sich offnet oder geöfnet wird, kann man eine ziemlich sichere Vermuthung haben, ob er virulent wird oder nicht. Geht das Hinschmelzen des Drüsengewebes schnell vor sich, ist wenig Empfindlichkeit, nicht besonders intensiver Rubor der Haut über demselben vorhanden, kann man mit ziemlich grosser Sicherheit die Diagnose nicht virulenter Bubo stellen. Macht man Probepunction und hat der ausgezogene Eiter ein grauliches, halb schleimiges A ussehen, kann man ohne noch Bacillen zu suchen, mit Sicherheit den Fall als nicht virulent ansehen. Nach dem Oeffnen zeigen diese Bubonen auch ein charakteristisches Verhalten. Die Secretion nimmt schnell ab. Die Wundränder werden nicht angefressen und heilen oft sehr schnell, oft innerhalb 8 Tagen. Ausmahmsweise kann bei scrophulösen Patienten die Drüsenanschwellung sich sehr lange halten und die Theilung lange Zeit in Anspruch nehmen mit Bildung ron Senkungen und Fisteln.

Ein grosser Theil der nicht virulenten Bubonen werden resorbirt, ohne dass sie sich öffnen oder geöffnet werden, was nie mit den virulenten geschehen kann.

Entwickelt sich dagegen $\operatorname{der} B u b o s c h n e l l$ mit grosser Empfindlichkeit und intensivem Ruborinder Haut, so kann die Entwicklung eines virulenten $B$ ubos befürchtet werden. Wenn er nicht incidirt wird, wird er sich schnell durch die Haut fressen, während die nicht virulenten sehr 
lange stehen können ohne durchzubrechen, selbst wenn die Haut über denselben sehr dünn ist.

Der Buboinhalt unterscheidet sich auch vom Inhalt des nicht virulenten Bubos, indem er gewöhnlich eine schmutzige Chocoladenfarbe besitzt.

Wenn ein solcher Bubo durch Incision geöffnet ist, wird

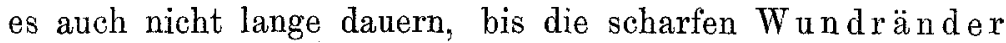
angefressen werden und ein charakteristisches chancröses Aussehen annehmen. Mit Rücksicht auf die Dauer der Behandlung besteht auch ein grosser Unterschied zwischen den nicht virulenten und den virulenten Bubonen.

Während die ersteren gewöhnlich (wenn sie nicht mit Scrophulose complicirt werden) überaus schnell nach dem Oeffnen zuheilen, ja sogar nicht selten, selbst nachdem das Drüsengewebe in grosser Ausdehnung weich geworden, zurückgehen ohne sich zu öffnen, nimmt die Behandlung der virulenten stets ziemlich lange Zeit in Anspruch, bei beliebiger Behandlung.

Wenn diese Patienten den ganzen Tag gehen oder stehen sollen, wie bei mehreren meiner obenerwähnten Fälle, zieht die Behandlung sich selbstredend etwas mehr in die Länge, als wenn sie im Hospital behandelt werden oder sich absolut ruhig verhalten; aber unter allen Umständen erfordert die Behandlung eine ziemlich lange Zeit.

Wenn in der Literatur verschiedene Mittheilungen über schnelle, zum Theil abortive Behandlungsmethoden für Bubonen erschienen sind, so ist nicht genügend Rücksicht darauf genommen worden, inwiefern der Bubovirulent war oder nicht.

Trifft man eine Serie nicht virulenter Bubonen und benutzt eine beliebige Behandlung, wird man stets glänzende Resultate erzielen.

\section{Behandlung.}

Nach meiner geringen Erfahrung scheint den virulenten Bubonen gegenüber die eine Methode nicht sonderlich besser als die andere zu sein. 
Wo man Jodoform gebrauchen kann, ist solches sicherlich das beste Mittel, aber ich habe es des Geruches wegen nur ausnahmsweise bei meinen Patienten gebrauchen können.

Ich habe nur grosse Incisionen und Auslöflung sowie Entfernung von unterminirten Hautpartien vorgenommen. $A b$ und zu Aetzungen mit rauchender Salpetersäure.

Verbinden mit Dermatol, Salicylwatte sowie Stärkebinde, die ein paar Mal wöchentlich gewechselt wurde.

Die rationellste und wirksamste Behandlungsmethode ist Welander's Wärmebehandlung, die auf dem Factum basirt ist, dass das Virus bei verhältnissmässig niedriger Temperatur ca. $40^{\circ}$ getödtet wird.

Leider erfordert diese Methode so viele Apparate, dass dieselbe nur für Hospitalsbehandlung passt.

Das Verhältniss der virulenten Bubonen kann somit in wenigen Zeilen resumirt werden:

1. Die Häufigkeit der virulenten Bubonen im Verhältnisszuden nicht virulenten scheintbedeutend zu variiren.

2. Die Virulenz wird vom Vorhandensein des Ulcus molle Bacillus bedingt.

3. Die Virulenz und somit auch der Bacillus sind gew öhnlich vor dem Oeffnen des Bubos vorhanden (bubon virulent d'emblée), können aber auch kommen, nachdem er geöffnet ist (bubon virulent consecutivement).

4. Die Differentialdiagnose zwischen virulentem und nicht virulentem Bubo kann im Allgemeinen mit ziemlich grosser Sicherheit gestellt werden, ehe derselbe ge öffnet wird.

Der klinische Unterschied $z$ wischen nicht virulentem und virulentem Bubo lässt sich folgendermassen schematisiren: 
Nicht virulenter Bubo.

Das Hineinschmelzen des Drüsengewebes geht langsam vor sich.

Geringe Empfindlichkeit. Wenig ausgesprochener Rubor der Haut über dem Bubo.

Der Inhait ist von graulichem, schleimigem Aussehen.

In demselben lassen sich keine $\mathrm{Mi}$ kroben nachweisen, weder mikroskopisch noch durch Züehtangsversuche.

Die Rundzellen färben sich schlecht.

Kann zurückgehen, ohne sich zu öffnen oder geöffnet zu werden.

Nach dem Oeffnen nimmt die Secretion schnell ab.

Die Wundränder nach der Incision werden nicht angefressen.

Die Heilung geht sehr schnell, oft innerhalb 8 Tagen.

\section{Virulenter Bubo.}

Das Hinschmelzen geht schnell vor sich.

Starke Empfindlichkeit. Intensiver Rubor.

Der Inhalt ist gewöhnlich schmutzig, chocoladenfarbig.

Der Ulcus molle-Bacillus lässt sich nachweisen, wennschon in spärlicher Anzahl.

Die Rundzellen färben sicb.

Geht niemals zurück, ohne sich zu öffnen oder geöffnet zu werden. Wenn er nicht incidirt wird, wird die Haut schnell von selbst perforirt.

Nach dem Oeffnen reichliche purulente Secretion.

Die Wundränder werden angefressen gleichwie die ganze Bubohöhle das Aussehen einer grossen Schankerwunde annimmt.

Die Heilung geht sehr langsam und nimmt oft Wochen und Monate. 